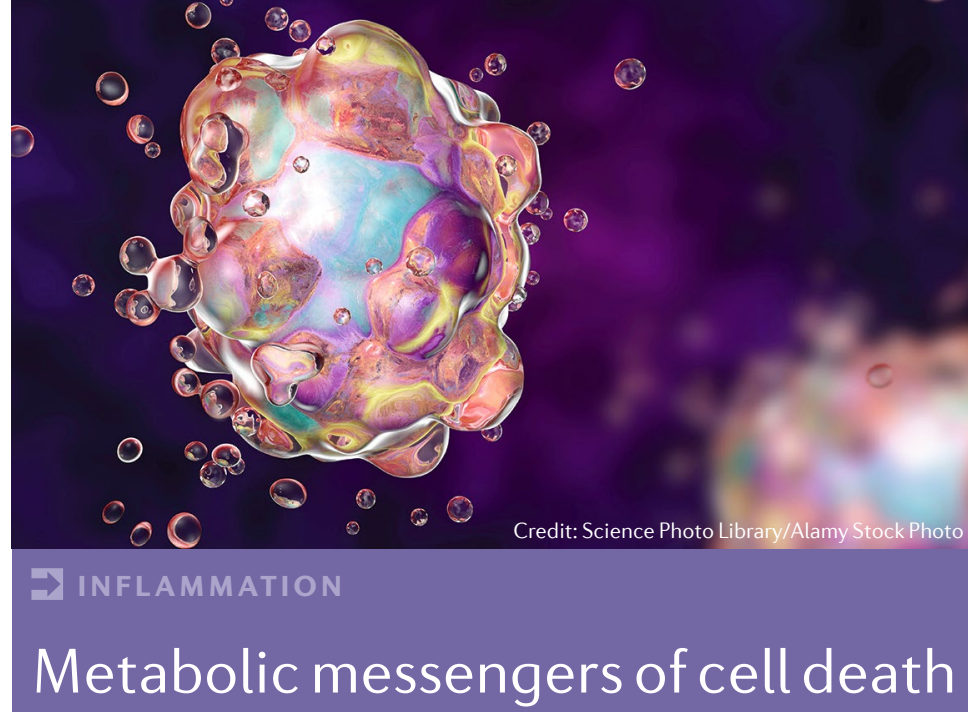

every 8 weeks improved pain and function but not PGA-OA, compared with placebo. Rapidly progressive OA occurred more frequently in the tanezumab $5 \mathrm{mg}$ group than in the tanezumab $2.5 \mathrm{mg}$ group (2.8\% vs $1.4 \%$ ).

ORIGINAL ARTICLE Berenbaum, F. et al. Subcutaneous tanezumab for osteoarthritis of the hip or knee: efficacy and safety results from a 24-week randomised phase III study with a 24-week follow-up period. Ann. Rheum. Dis. https://doi.org/10.1136/annrheumdis2019-216296 (2020)

\title{
$\Rightarrow$ GOUT
}

\section{Hospitalization for infection on the rise in gout}

Serious infections were the reason for $14.5 \%$ of all hospitalizations among people with gout in the USA in 2015-2016, compared with $8.9 \%$ in $1998-2000$, according to an analysis of data from the U.S. National Inpatient Sample. From 1998 to 2016, rates of hospitalization due to sepsis increased 19.3-fold, for opportunistic infections increased 2.6-fold and for pneumonia increased 2.2-fold. The overall rate of in-hospital mortality was $10.1 \%$ for those with sepsis, $4 \%$ for opportunistic infections, $3.1 \%$ for pneumonia, $0.6 \%$ for skin and soft tissue infection and $0.5 \%$ for urinary tract infections.

ORIGINAL ARTICLE Singh, J. A. \& Cleveland, J. D. Serious infections in people with gout in the United States: a national study of incidence, time-trends and outcomes. Arthritis Care Res. https://doi.org/10.1002/ACR.24201 (2020)

\section{$\Rightarrow$ OSTEOARTHRITIS}

\section{Physical therapy better than steroids for knee OA}

In a randomized trial $(n=156)$, patients with clinical and radiographic evidence of knee osteoarthritis $(\mathrm{OA})$ who underwent physical therapy had less pain, stiffness and functional disability than patients who received an intraarticular glucocorticoid injection, as measured by total WOMAC score at 1 year (37.0 vs 55.8 points; mean betweengroup difference 18.8 points; $95 \% \mathrm{Cl}$ 5.0-32.6). Similar differences were seen in the secondary outcomes, which included the results of two functional tasks and the Global Rating of Change scale score, all assessed at 1 year.

ORIGINAL ARTICLE Deyle, G. D. et al. Physical therapy versus glucocorticoid injection for osteoarthritis of the knee. N. Engl.J. Med. 382, 1420-1429 (2020)

\section{$\Rightarrow$ VASCULITIS}

\section{GCA relapse common in ustekinumab trial}

IL-12-IL-23 blockade therapy with ustekinumab, in combination with a 24-week prednisone taper, was associated with a high rate of treatment failure in patients with active new-onset or relapsing giant cell arteritis (GCA) in a prospective, open-label trial. The study enrolled 13 patients, all of whom achieved disease remission within 4 weeks of starting treatment but only three (23\%) of whom achieved the primary end point of sustained, prednisone-free remission (defined as the absence of relapse through week 52). Enrolment in the trial was terminated early, after seven of the first ten patients recruited experienced GCA relapse.

ORIGINAL ARTICLE Matza, M. A. et al. Ustekinumab for the treatment of giant cell arteritis. Arthritis Care Res. https://doi.org/10.1002/ACR.24200 (2020)

Apoptosis, a form of programmed cell death, can modulate various physiological processes including inflammation, the dysregulation of which can lead and/or contribute to various pathologies such as inflammatory arthritis. New findings show that during cell death, and while maintaining their membrane integrity, apoptotic cells can release metabolites that can influence the surrounding environment, including having anti-inflammatory effects.

"It has been a long standing observation in the field that apoptosis, unlike other forms of cell death, is anti-inflammatory, and can also have other beneficial effects such as inducing compensatory proliferation of cells in the tissue, as well as tissue repair," explains corresponding author Kodi Ravichandran. "What has long been unknown is what makes the apoptotic process capable of these features."

Although previous studies had identified a number of apoptotic cellderived soluble factors that function as 'find-me' signals to attract phagocytes, the full apoptotic secretome had yet to be characterized. In the new study, the researchers used a combination of metabolomics and transcriptomics to identify the factors released from apoptotic cells that might influence the surrounding tissue microenvironment. "We integrated data across different cell types and different modalities of cell death induction, using metabolic flux analysis, as well as a combination of in vitro and in vivo approaches and disease models, to comprehensively address the question of 'how do apoptotic cells communicate with their neighbourhood," reports Ravichandran.

They identified a group of metabolites released from apoptotic cells that could induce specific gene expression programmes in healthy neighbouring phagocytic cells, including genes involved in cytoskeletal rearrangements, inflammation, wound healing or tissue repair, anti-apoptotic functions, metabolism and cell size regulation.

"By comparing the metabolites released from primary cells and cell lines with or without apoptotic stimuli, as well as characterizing the metabolites released via a specific membrane channel that gets activated during apoptosis (the pannexin 1 channel), we could narrow the list of metabolites down to six or even three with beneficial properties," adds Christopher Medina, the first author of this work.

A cocktail of these three or six metabolites (termed Memix-3 or Memix-6, respectively) could alleviate inflammation and paw swelling in mice with $\mathrm{K} / \mathrm{BxN}$ serum-induced arthritis. Notably, treatment with the Memix-3 cocktail could also protect the joints of the mice from inflammation, bone erosion and cartilage erosion.

"We hope these results advance the knowledge in the field in terms of considering metabolites, metabolism and cellular communication," says Ravichandran. "Going forward, we are very interested in examining the mechanisms by which the apoptotic metabolites signal to the surrounding cells, as there is quite a bit we don't know, such as what receptors are used by these metabolites, or whether the metabolites are passing through the membranes, and how they work jointly to produce their effects." Jessica McHugh

ORIGINAL ARTICLE Medina, C. B. et al. Metabolites released from apoptotic cells act as tissue messengers. Nature 580, 130-135 (2020) 